\title{
Interferometric fringes projection system for 3D profilometry and relief investigation.
}

\author{
Vincent Moreau $^{\mathrm{a}}$, Dimitri Laboury ${ }^{\mathrm{b}}$, Bernard Tilkens ${ }^{\mathrm{a}}$, Yvon L. M. Renotte ${ }^{\mathrm{a}}$ \\ ${ }^{a}$ Hololab, Dept. of Physics, University of Liège, Allée du 6 Août, Bat B5, B-4000 Liège (Belgium) \\ ${ }^{\mathrm{b}}$ Egyptological Dept. and European Center for Archaeometry of the University of Liège, quai \\ Roosvelt 1B, B-4000 Liège (Belgium)
}

\begin{abstract}
We present a new 3D full-frame profilometer based on structured laser light projection method. This device takes advantage of the polarization states splitting technique for producing and shifting multiple sinusoidal Young's interference patterns that are projected on the inspected surface. The principle of the technique is presented and we discuss the advantages of monochromatic light projection method as a mean to overcome ambient lighting for in-situ measurement. Some results that we obtained on objects from the Egyptian Department of the British Museum are presented to demonstrate that $3 \mathrm{D}$ laser profilometry is a worthwhile technique for epigraphic investigations where naked-eye inspections fail.
\end{abstract}

Keywords: structured light, profilometry, 3D measurement, archeometry

\section{INTRODUCTION}

Non-contact optical measurement methods are essential tools in many industrial and research domains. They are commonly applied to extract the range data of surfaces, from nanometer to kilometer scale. The projected-fringes techniques are among the preferred approaches for measuring shape, surface profile and displacement of usual size objects. They allow robust, precise and fast whole-field acquisitions. Furthermore they benefit of the well-established procedures developed for interferometric systems such as phase-shifting ${ }^{[1]}$ and phase unwrapping algorithms ${ }^{[2]}$.

In a typical way, one or multiple structured light patterns are projected on the surface to be analyzed. They are usually characterized by a periodic variation of the intensity in such a way that a specific phase can be associated to every enlightened points of the object. By recording the scene with a CCD or CMOS camera, it is possible to compare the phase distribution of the image points to the linearly growing phase of a non-distorted grid. This phase difference contains information required for a computation of the surface height variations based on triangulation formulas.

The required features for a good projection pattern are: a perfect sinusoidal irradiance function, a very high contrast, high irradiance and a large depth of field. Conventional projection technologies such as Spatial Light Modulator (SLM) or slide projector hardly ever satisfy all these qualities. The problem of contrast is especially critical when ambient light cannot be shut down, for instance in outdoor conditions.

An attractive alternative to SLM is given by interferometric fringe projection systems ${ }^{[3]}$. Young's interference pattern is a theoretically perfect sinusoid that can have a very high contrast. In addition, interferometric fringes are non-localized which means that the irradiance function and contrast remain unchanged whatever the projection distance so there is no depth field problem. The use of monochromatic laser light is also a beneficial approach for filtering relevant signal from ambient light.

Compared to SLM, dynamically shifting or scaling interferometric projection pattern could be a real challenge. It often requires precise and complex electromechanical or optoelectronic systems whose repeatability and robustness is not assured. Internal vibrations are also a possible cause of trouble that compromises fringes stability.

In this paper we present an original monolithic device that allows fringes generation and phase-shifting without any moving part. Simplicity, robustness, insensitivity to vibrations and low-cost are among the principal qualities of this 
setup. We also show that it is possible to create a large variety of pattern fringes spacing for multiple ranges measurement and temporal phase unwrapping ${ }^{[4]}$.

In the first section, we bring up the theoretical bases of relief analyses by fringes projection and remind the concept of phase shifting and temporal unwrapping. In the second section we present the polarization based splitting setup that enables practical implementation of the dynamic functions. We also focus on the practical image enhancements allowed by the physical properties of polarized laser light.

Section four is devoted to some interesting results that we obtain on several Egyptian antiquities from the British Museum collections. They highlight the scientific contribution that such device can bring to historical investigation.

\section{DYNAMIC FRINGES PROJECTION METHODS}

\section{From phase to 3D coordinate}

Mathematically, the intensity of point $\mathrm{P}(\mathrm{x}, \mathrm{y}, \mathrm{z})$, recorded at the $(\mathrm{u}, \mathrm{v})$ camera pixel is represented by the following expression :

$$
I(u, v)=I_{0}(u, v)[1+V(u, v) \cos \varphi(u, v)]
$$

where $I_{0}$ is the background intensity distribution, generally correlated with the object diffusion distribution; $V$ is the local fringes contrast (ranging from 0 to 1 ) and $\varphi$ is the phase information which can be related to the distance of the object point $\mathrm{P}$ by using the next treatment.

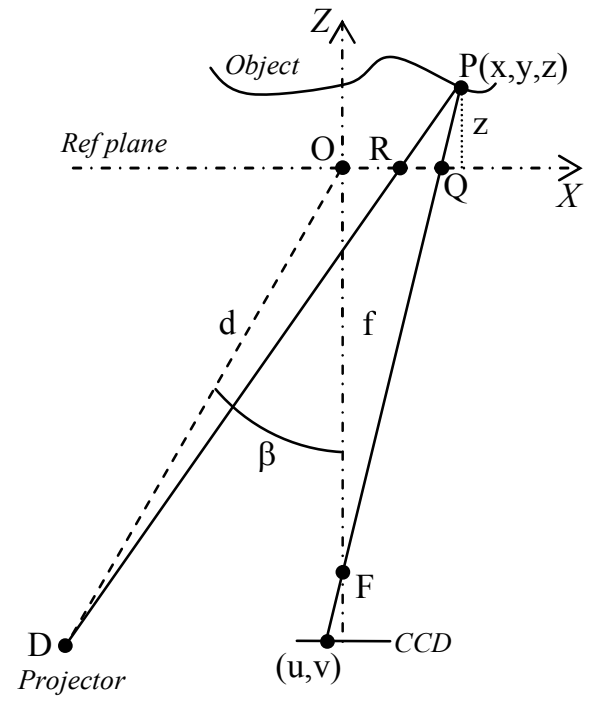

Figure 1 : Triangulation scheme for fringes projection profilometry.
Let us consider the point $\mathrm{O}$, at the intersection of the optical axes of the projector and the camera that form an angle $\beta$. Distance between $\mathrm{O}$ and the focal points of projector and camera are noted $\mathrm{d}$ and $\mathrm{f}$ respectively. $\mathrm{O}$ is the origin of a reference system with $\mathrm{X}$ and $\mathrm{Y}$ axis perpendicular to the optical axis of the camera and parallel to detector rows and columns. The camera pixel $(\mathrm{u}, \mathrm{v})$, image of $\mathrm{P}$, is related to a point $\mathrm{Q}$ in the reference plan OXY, which coordinates depend on the product of $\mathrm{f}$ distance and a scale factor $\mathrm{G}$.

$$
x_{Q}=\left(u-\frac{N}{2}\right) \cdot G \cdot f
$$$$
y_{Q}=\left(\frac{M}{2}-v\right) \cdot G \cdot f
$$

Equ. 2

where $\mathrm{N}$ and $\mathrm{M}$ are the number of pixel by row and column respectively.

Expressing that $\mathrm{P}$ is the intersection of $\mathrm{DR}$ and $\mathrm{FQ}$ gives:

$$
\begin{aligned}
& z=\frac{f \cdot d \cos \beta\left(x_{R}-x_{Q}\right)}{f\left(d \sin \beta+x_{R}\right)-x_{Q} d \cos \beta} \\
& x=\frac{(f-z) x_{Q}}{f} \\
& y=\frac{(f-z) y_{Q}}{f}
\end{aligned}
$$

Equ. 3

The problem is reduced to find the coordinate of point $\mathrm{R}$ of the undistorted grid pattern which phase is the same than the phase in $\mathrm{P}$ (and Q).

$$
\varphi_{R}=2 \pi \frac{x_{R} \cos \beta}{\Lambda\left(d+x_{R} \sin \beta\right)}
$$


where $\Lambda=\frac{\lambda}{a}$ is the projected fringe nominal period for a Young interference with source distance $a$ and wavelength $\lambda$.

Noting that $\varphi_{R}=\varphi_{Q}$ yields

$$
x_{Q}=\frac{\Lambda \cdot d \cdot \varphi(u, v)}{(2 \pi \cos \beta-\Lambda \cdot \varphi(u, v) \cdot \sin \beta)}
$$

Substituting equations 2 and 5 in system 3 yields the full 3D coordinates of every image point where phase can be unambiguously determined.

\section{Phase-shifting profilometry}

Many different methods exist to evaluate the phase associated to each pixel of a projected fringes pattern. We can clearly distinguish the single-frame methods used in Fourier Transform Profilometry ${ }^{[5]}$ (FTP) and Wavelet Transform Profilometry ${ }^{[6]}$ (WTP), and multi-frame methods as Temporal Phase-Shifting ${ }^{[4,7]}$ (TPS) and Spatial Phase-Shifting ${ }^{[8]}$ (SPS). The formers are particularly appreciated for they high speed and real-time potential while the seconds are among the best ways to recover the phase with high accuracy.

Temporal Phase Shifting Profilometry is subject to regular innovations and developments. In general phase-shifting method, a fixed number of images are recorded with a gradual global phase increment between each:

$$
I_{n}(u, v)=I_{0}(u, v)\left[1+V(u, v) \cos \left[\varphi(u, v)+\Delta_{n}\right]\right] \quad n=0, . ., N
$$

Equ. 6

For $N \geq 3$ the phase $\varphi(u, v)$ can be analytically resolved independently of $I_{0}$ and $V$. However one knows that the quality of solution is hardly conditioned by the precise determination of phase increment $\Delta_{n}$. Additional phase shifted images can be a solution when increment uncertainties have a specific structure, i.e. a systematic linear or quadratic error. When phase-shift error are unpredictable, automatic calibration based on frequency-domain analyze of each image ${ }^{[9]}$ can provide a valuable correction.

\section{Multi-resolution profilometry}

Whatever the technique implemented for extracting phase from fringes patterns (Fourier or phase shifting), it always produces a wrapped phase map, i.e. phase value lying in the range $[-\pi, \pi]$. The restoration of the unknown multiple of $2 \pi$ to all pixels is the object of many automatic phase unwrapping algorithms, usually based on spatial comparison of phase value at neighboring pixels. Anyway, some complex object topologies, i.e. isolated regions, remain indefinite or uncertain. That is the reason why several techniques have been developed in recent years for the temporal evaluation of phase maps, allowing independent recovering of the phase of each pixel. The basic idea is to vary the pitch of the fringes over time, getting from a low frequency pattern, on which no ambiguous phase shift occurs, to a high frequency one able to give a high precision result. The phase at each pixel is then unwrapped along the time axis and the phase value on large fringes are used to find the phase order of the small ones.

\section{POLARIZING BEAM SPLITTER FOR DYNAMIC INTERFERENCE PATTERN PROJECTION}

Spatial and amplitude splitting of a wavefront are the two ways for producing stable interference patterns. Young and Lloyd interferences are common examples of spatial division while Michelson or Fabry-Perot interferometers illustrate the amplitude division technique.

Polarization state splitting method is a particular case of amplitude division in which the transmitted and reflected waves have orthogonal polarization. Such effect can be observed in birefringent crystal placed between crossed polarizers and find many applications in interferometry ${ }^{[10]}$. 
Polarization division is hardly advantageous to build common path interferometers as the two arms are always distinguishable even when beams are superimposed.

The common path dynamic fringes projector that we develop is schematized on figure 2 . The key element is a glass prism coated on its hypotenuse with a Bragg grating. It is well known that under a particular incidence, i.e. Brewster angle, Bragg gratings are polarization selective, which means that transverse electric (TE) polarization state is reflected and transverse magnetic (TM) state is transmitted. A $532 \mathrm{~nm}$ beam produced by DPSS laser is polarized at $45^{\circ}$ relatively to the incidence plan and focused on the grating. The TE component is reflected and the TM polarization mode is transmitted and undergoes total internal reflection on the external interface of a planar glass plate bond to the prism. This process creates two orthogonally polarized spherical waves that propagate in the same direction but that are spatially sheared by a distance $\sqrt{2} d$, where $\mathrm{d}$ is the thickness of the planar substrate. Then, these two shares pass through a liquid crystal variable retarder whose fast and slow axes are parallel to the TE and TM axes respectively. Depending on the voltage applied to the retarder, an adjustable phase shift is introduced between TE and TM waves. At the output of the system, close to the casting lens, a second linear polarizer, used as an analyzer, makes the interference fringes visible by projecting TE and TM mode on a common oscillation plane.

As the fringe spacing of the projected pattern depends on the thickness of the planar substrate, multiple range measurements are possible by using a multi-step substrate and by moving the prism up and down.

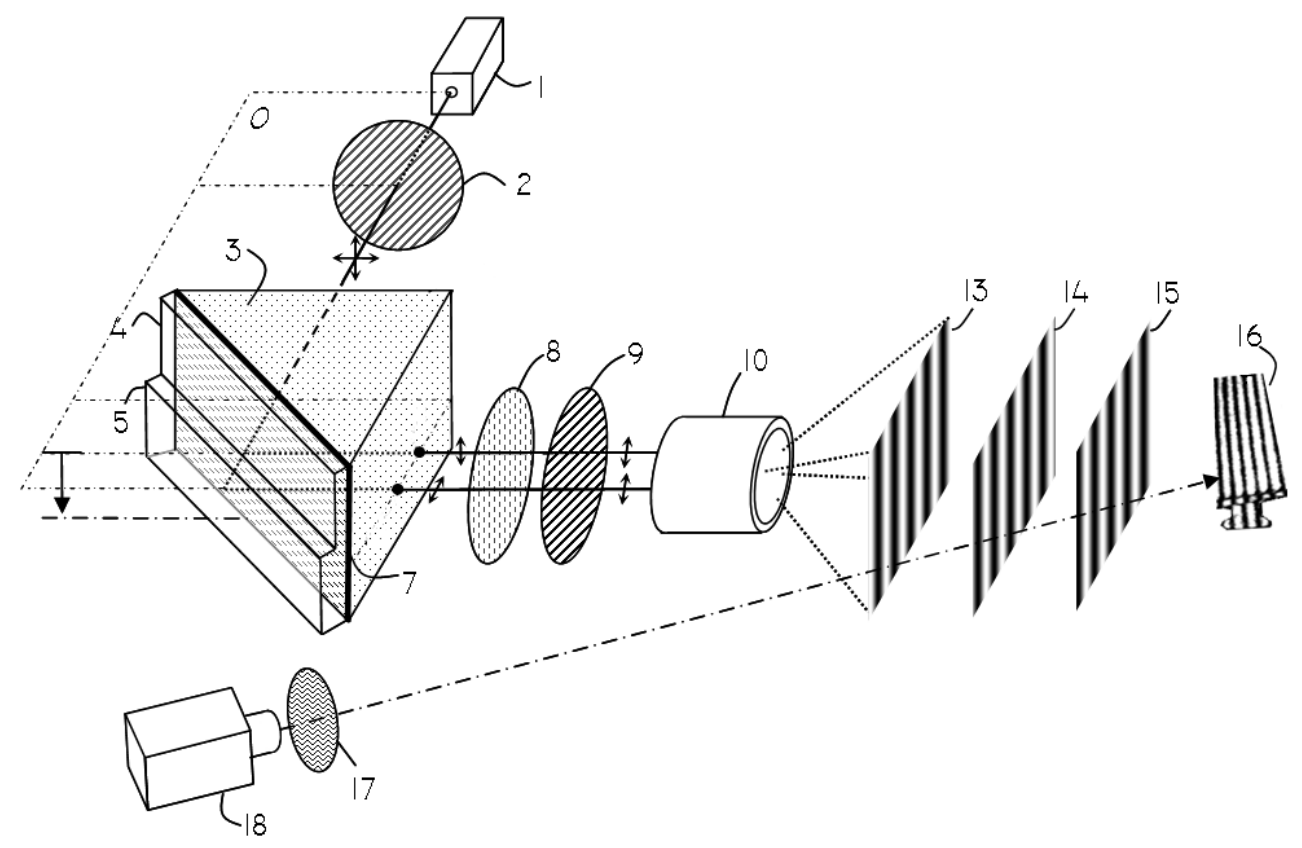

Figure 2 : Optical setup for multi-resolution laser fringes projection system : 1-laser source; 2,9-linear polarizer; 3-prism; 4,5-multistep glass substrate; 7-polarizing Bragg grating; 8-liquid crystal retarder; 10-projection lens; 13,14,15-phase shifted patterns; 16-object; 17-camera filter; 18-digital camera.

In front of the acquisition camera, different filters can be set in order to improve the images quality. The most frequent cause of signal disturbance is the ambient light. Classical structured light projection systems are unable to work correctly under daylight conditions because of the poor fringes contrast. Laser light has the advantage of a very narrow spectral band $(<1 \mathrm{~nm})$. Using an interferential spectral filter adapted to the laser frequency one can reduce drastically upsetting light while preserving the fringes image.

Specular or partially specular objects are also known to be especially difficult to digitalize because of the saturation effect due to the rays that are directly reflected to the camera. Polarization properties of the projected laser fringes can help us to overcome these specular reflections. Indeed polarization state of a ray is preserved by specular reflection 
whereas diffused rays are depolarized. A linear polarizer upstream the camera lens then absorbs all specular ray and transmit 50 percents of the diffused light coming from the object, thus resolving the problem caused by saturation. Figure 3 illustrates these enhancement allowed by joint use of laser fringes projection and image filtering.
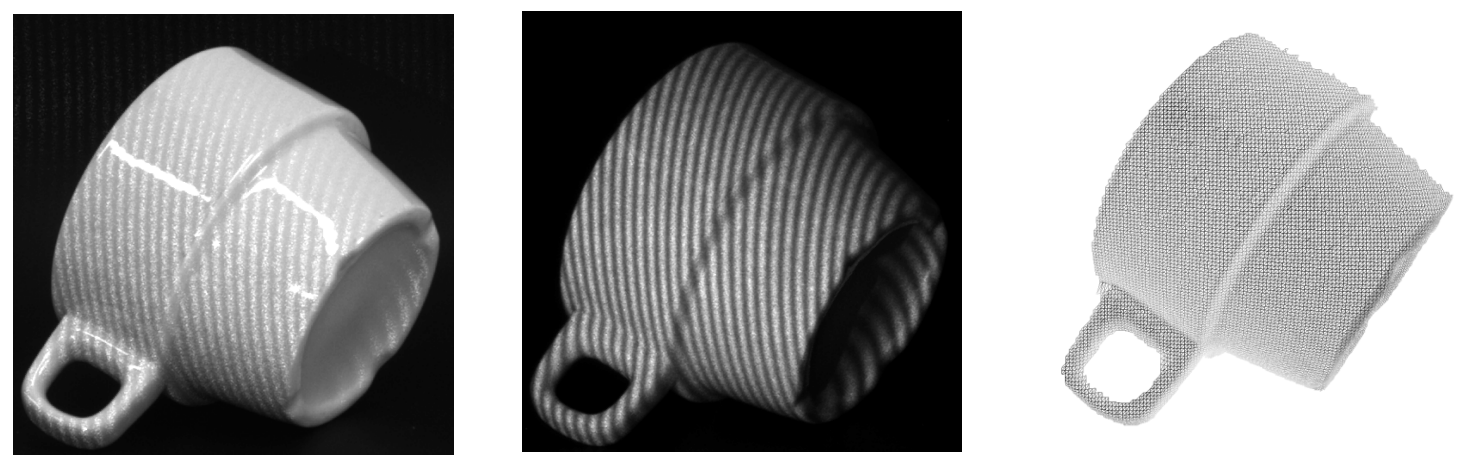

Figure 3: Contrast restoration and saturation reduction by spectral an polarization filtering: a) projected fringes under ambient lightening without filtering; b) image restoration by filtering; c) 3D object reconstruction from filtered images.

\section{ARCHAEOLOGICAL SURVEYES}

The recordings needed by the different aspects of archaeological research have a double goal: the creation of a medium allowing to publish in the more accurate way the studied object; and, even more importantly, the virtual conservation of this object, or, at least, the conservation of the historical information it reveals and preserves.

Until now, to fulfill these purposes, archaeologists and cultural Heritage specialists usually rely upon recording techniques based on photography and handmade drawing. But these traditional techniques raise serious problems of accuracy, objectivity, speediness and flexibility of realization and use.

New optical inspection techniques allow recording and processing the information that defines the precise 3D shape of an object.

We present hereafter interesting results obtained on monuments from the Egyptian Department of the British Museum (London). These examples demonstrate that the structured laser light profilometer is a powerful tool, not only for preservation, but also for scientific investigation of features that remain almost unrevealed by visual inspection.

\section{Geometry versus texture}

A 3D recording allows distinguishing and isolating the real geometry of an object from its purely chromatic characteristics, two features that are always merged in our ocular perception of reality. It is often interesting to map the chromatic or texture information on the recorded geometry of a digitalized object, but in some cases, it might also be very useful to separate these two material characteristics of the object.

Figure $4 \mathrm{a}$ is a classical photograph of an inscription on a fragment of an ancient Egyptian naos or stone tabernacle for a cult statue (BM EA 1106). The colors of the stone - Egyptian pink granite - and the damages caused to the monument make the inscription barely readable. On figure $4 \mathrm{~b}$ a grey scale height map shows the same portion of the stone in a purely geometrical representation without any chromatic information or, in this case, disturbance. Every hieroglyph becomes now easily readable and the specialist can easily recognize the royal title and name of the last indigenous Pharaoh of Egypt, king Nectanebo II. Since the engraving of this inscription is intentional, it is situated almost at the same depth from the surface of the stone and appears in the same grey level. On the contrary the damages have necessarily variable depth and looks like clouds, with variable grey value. 

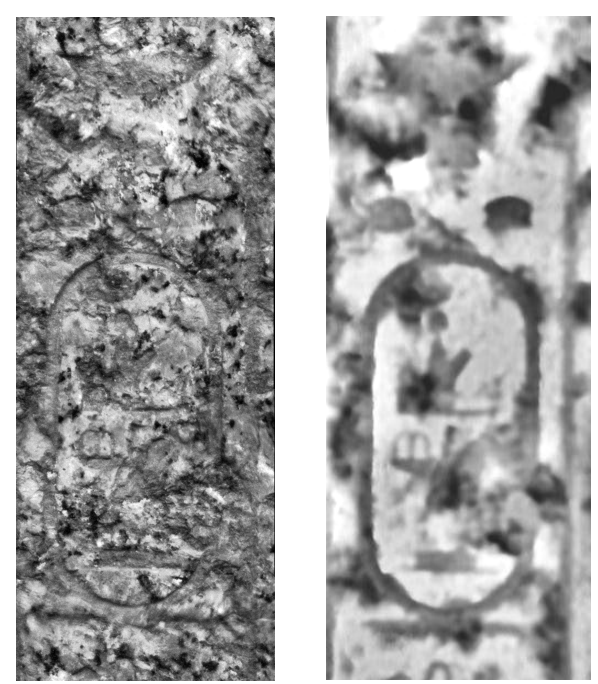

Figure 4 : 3D digitalization of a damaged hieroglyphic inscription on an ancient Egyptian granite monument (BM EA 1106) : a) photography ; b) Grayscale height map revealing the hieroglyphs.

\section{In situ digitalization}

The ancient Egyptian head presented on figure 5 is a part of a large bas-relief depicting the prince Rahotep, a son of king Kheops, the famous builder of the greatest pyramid at Guiza, who lived more than 45 centuries ago. This commemorative monument is currently on display in the upper galleries of the British Museum (BMEA 1242). It was digitalized in normal museum working condition, which is in the public galleries, with a natural and varying ambient daylight and through its protective glass. The first picture is a grayscale height map very common and useful to isolate tiny relief variation, analysis and interpretation of digitalized item. The right graph is a depth expended transversal section of the head that reveals a very slight slope of the surface of the worked stone at the approach to the face of Rahotep. This tiny depression, which enhances the sculpted motif, is almost imperceptible through a visual inspection of the relief.
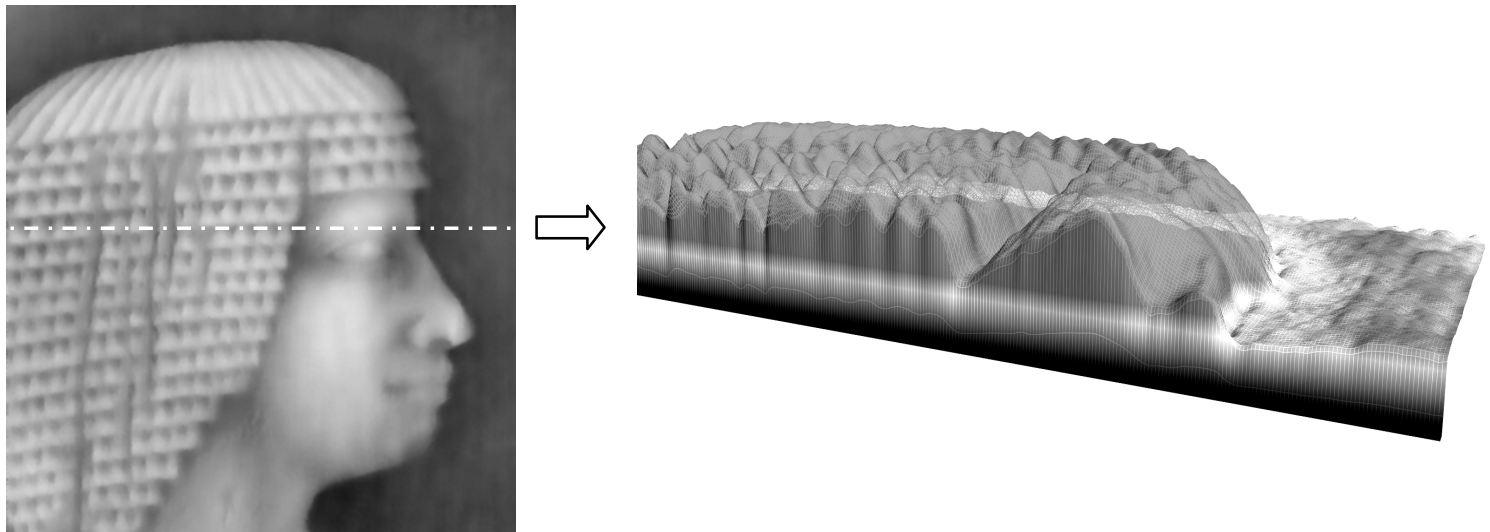

Figure 5 : 3D digitalization of a detail of the Rahotep slab-stele BM EA 1242. a) Greyscale heightmap; b) Depth expanded representation of a slide. 


\section{Real 3D reading}

Some archaeological artifacts are in such a state of preservation that it is almost impossible to handle them without damages. It is precisely the case of this ancient Egyptian stele, an almost 4000 years old funerary monument now in the British Museum (BM EA 224). It was carved in a poor quality limestone and, because of its old age, is nowadays decaying,-even in the particularly excellent conservation conditions of one of the greatest Museums in the world.

The central picture is a photograph of the stele with good low-angled light in order to let the very faint relief of the decoration be visible. Even in these ideal conditions, the carvings are not easily recognizable. The extracted detail below shows a part of the rather difficult to read hieroglyphic inscription. The image on the left is an old traditional handmade copy produced by drawing in order to publish a readable representation of the stele. The same extracted detail exemplifies the contour line drawing technique usually used to suggest the relief. The right picture is a partly completed 3D capture of the stele made by our laser fringes projector. The extracted detail now shows new informations : on the right-hand side, the two signs on the left on the two leg hieroglyphs, one on top of the other, are not just simple strokes, as recorded on the previously published handmade drawing, but appear to be reed leafs, i.e. another hieroglyph that changes the reading of the personal name referred to the inscription.

The 3D recording is obviously much more accurate, reliable and, thus, useful than the traditional drawing, even if the latter was made long ago, at a time when the object was much better preserved. Furthermore it allows free using and manipulating of the virtual object as if it was the real one, but without any risk for the latter.
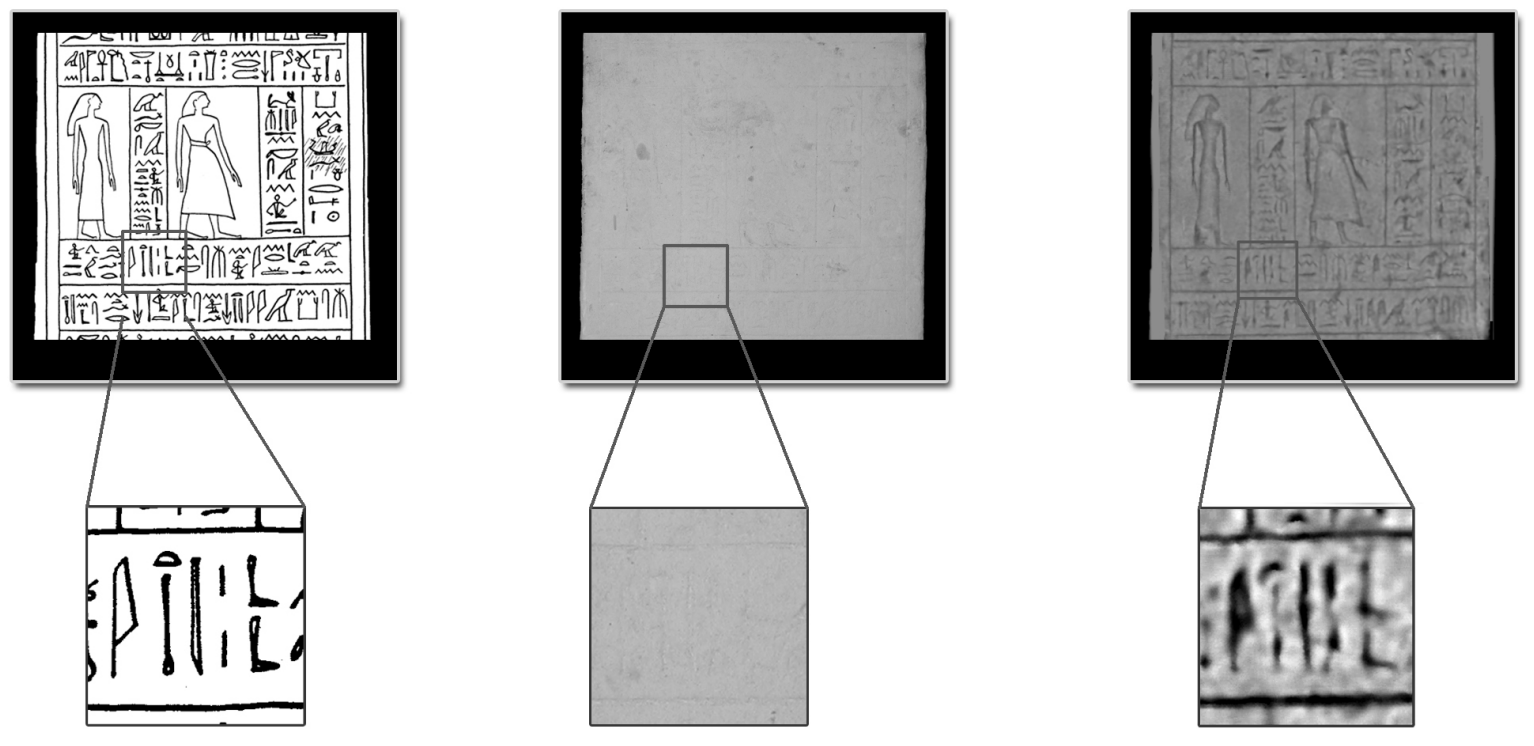

Figure 6 : 3D digitalization of an ancient Egyptian stele BM EA 224 : a) published traditional handmade drawing; b) picture under grazing incident lightening; c) grayscale height map. 


\section{CONCUSION}

The projection and the reading of fringes patterns are realized by an original setup based on reconfigurable and dynamic laser light interference. This completely portable device is specifically dedicated to the quick acquisition and accurate numerical 3D recording of archeological documents. We show that the physical properties of polarized laser light make possible the capture of partially specular object, even in daylight conditions or behind a protective glass, without texture disturbance. With this technique, many archeological artifacts can be digitalized, analyzed, measured and even duplicated virtually or physically. Thus the accurate and precise 3D representation of the endangered historical traces will be able to remain undamaged across time and space.

\section{ACKNOWLEDGMENT}

It is a pleasure to thank the following institutions for their financial and scientific support of the OSIRIS project (Optical Systems for Interferometric Relief Investigation and Scanning):

- The Communauté Française of Belgium (Action de Recherche Concertée entiltled "Pour une histoire matérielle de l'Art")

- The Région Wallonne (DGTRE-First Spin off project)

- The British Museum and especially its Egyptian Department.

- DEIOS s.a., Wallonia Space Logistic, Liege Sciences Park

Our attendance at the Munich conference was made possible thanks to the support of the Communauté Française of Belgium.

\section{REFERENCES}

1. D.W. Robinson and G.T. Reid, Interferogram Analysis : Digital Fringe Pattern Measurement Techniques, Institute of Physics Publishing, Bristol and Philadelphia, (1993)

2. D.C. Ghiglia and M.D. Pritt, Two-Dimensional Phase Unwrapping: Theory, Algorithms and Software, Wiley eds., New York (1998)

3. F. Wu, H. Zhang, M.J. Lalor and D.R. Burton, A novel design for fiber optic interferometric fringe projection phase-shifting 3-D profilometry, Optics Communications 187 (2001) 347-357

4. J.M. Huntley and H. Saldner, Temporal phase unwrapping algorithm for automated interferogram analysis, Appl. Opt. 17 (1993) 3047-3052

5. M. Takeda, H. Ina and S.Kobayashi, Fourier-Transform Method of Fringe-pattern Analysis for Computerbased Topography and Interferometry, JOSA 72 (1981) 156

6. J. Zhong and J. Weng, Spatial carrier-fringe pattern analysis by means of wavelet transform: wavelet transform profilometry, Appl. Opt. 43 (2004), 4993-4998

7. C.E. Towers, D.P. Tower and J.D.C Jones, Generalized frequency selection in multifrequency interferometry, Opt. Lett. 29 (2004), 1348-1350

8. S. Nakadate and M. Isshiki, Real-Time vibration measurement by spatial phase-shifting technique with a tilted holographic interferogram, Appl. Opt. 36 (1997), 281-284

9. K.A. Goldberg and J. Bokor, Fourier-transform method of phase-shift determination, Appl. Opt. 40 (2001) 2886-2894

10. B.L. Van Horn and H.H. Winter, Analysis of the conoscopic measurement for uniaxial liquid-crystal tilt angle, Appl. Opt. 40 (2001) 2089-2094 